\title{
Prospective Study on the Treatment of Mild Hypertension in the Aged
}

\author{
Kizuku Kuramoto, M.D., Satoru Matsushita, M.D., \\ Iwao Kuwajima, M.D., and Mototaka Murakami, M.D.
}

\section{SUMmARY}

The 4 year prospective trial on the effectiveness of the antihypertensive treatment was performed in 100 mild hypertensive patients of the aged, the average age being 76.1 years. Dropouts during the drug-off control period were 9 cases. The matched pair group was selected by the age, sex, and blood pressure. Forty-four drug treated cases and 47 placebo treated cases were comparable in blood pressure as well as in laboratory data.

Cerebrovascular and cardiac complications were observed in 4 cases or $10.5 \%$ in the drug group, and in 9 cases or $22.0 \%$ in the placebo group. When 8 cases of blood pressure elevation over $200 / 110 \mathrm{mmHg}$ in the placebo group were added to the cardiovascular complications, dropouts in placebo group reached $41.5 \%$, and this showed the significant difference. Other complications were observed in 12 cases or $31.6 \%$ in the drug group and in 17 cases or $41.5 \%$ in the placebo group. Major complications were cancers, infections, and bone or joint diseases.

Blood pressure was decreased from $171 / 87$ to $151 / 80$ in the drug group, and the average decrease was $20 / 7 \mathrm{mmHg}$ in 4 year period. No significant changes in hematocrit, serum protein, urea nitrogen, uric acid, sodium, and potassium were observed during the trial period. The present study suggested that antihypertensive treatment was effective in the aged with mild hypertension, and that careful follow up was needed not only for cardiovascular complications but also for general health condition.

\section{Additional Indexing Words :}

Prospective antihypertensive treatment Elderly hypertension Cerebro-cardiovascular complications Thiazide treatment

HERE have been dissenting views on the effectiveness of the antihyperten-
sive treatment of the mild hypertension in the elderly. The atherosclerosis of the cerebral or coronary artery has already been established in the elderly, and decrease in blood pressure reveals little benefit in the cerebral or coronary complications. ${ }^{1,2)}$ Furthermore, a marked reduction in blood pressure with an inappropriate antihypertensive therapy may critically decrease

From the Department of Medicine, Tokyo Metropolitan Geriatric Hospital, Tokyo.

Address for reprint: Kizuku Kuramoto, 35-2 Sakae-cho, Itabashi-ku, Tokyo 173, Japan.

Received for publication March 26, 1980. 
the cerebral or coronary blood flow, and induce ischemia of the target organs. ${ }^{3)}$ However, it is also known that hypertension in the elderly shows the higher prevalence of cerebral or cardiovascular complications, and the lower survival rate than the normotensive group. ${ }^{4), 5)}$

In order to evaluate the effectiveness of the long term antihypertensive treatment in the mild hypertension of the aged, prospective trial was performed by comparing the drug treated group with the placebo treated group of matched pair subjects.

\section{METHODS}

One hundred patients enrolled in the study were residents of the Tokyo Metropolitan Homes for the aged, and showed normal activity of daily living without complications. The previous antihypertensive medications were withdrawn and switched to the placebo, and the blood pressure reached a new level in a month. ${ }^{6)}$ The patients with blood pressure of more than $160 / 90$ and less than $200 / 110 \mathrm{mmHg}$ were included in the study. The matched pair group was selected by the age, sex, and blood pressure levels during the drug-off control period of about 1 year. During this control period 9 patients were dropped out because of blood pressure elevation in 6 , and one each of cerebral bleeding, cholelithiasis, and moving out. Thus, remaining 91 patients were divided into 44 of the drug treated group and 47 of the placebo treated group. Average ages of male patients in each group were 74.9 and 75.7 years, and those of female patients were 76.3 and 77.9 years respectively (Table I).

Patients were followed up once or twice a month for 4 years from 1975 to 1979. Patients were excluded from the trial when the blood pressure exceeded 200/110, and appearance of cerebrovascular or cardiac complications, other diseases which needed hospital admission, death, or moving out from the Home were considered to be the dropout.

In the drug treated group thiazide diuretics (trichlormethiazide 1 to $4 \mathrm{mg}$ ) were used as basal antihypertensive drugs. Thiazide monotherapy was used in 35 cases out of 44 cases. In the remaining 9 cases reserpine $(0.3 \mathrm{mg})$, methyldopa (125 to $500 \mathrm{mg}$ ), and hydralazine ( 50 to $100 \mathrm{mg}$ ) were added by stepped care approach when needed.

Table I. The Age and Sex Distribution of the Subjects

\begin{tabular}{cc|c|c|c|c|c|c}
\hline \multicolumn{2}{c|}{ Age } & 60 & 70 & 80 & 90 & Total & Average age \\
\hline \multirow{2}{*}{ Drug } & M & 5 & 14 & 4 & 0 & 23 & $74.9 \pm 5.4$ \\
& F & 0 & 16 & 5 & 0 & 21 & $76.3 \pm 4.6$ \\
\hline \multirow{2}{*}{ Placebo } & M & 4 & 15 & 6 & 2 & 27 & $75.7 \pm 7.3$ \\
& F & 3 & 9 & 7 & 1 & 20 & $77.9 \pm 6.7$ \\
\hline \multicolumn{2}{c|}{ Total } & 12 & 54 & 22 & 3 & 91 &
\end{tabular}




\section{Results}

Blood pressure and laboratory data in the control period:

The blood pressure level and laboratory data during the control period are shown in Table II. Average blood pressures were 166.1/85.5 $\pm 4.3 / 2.2$ $\mathrm{mmHg}$ in the placebo group, and $171.3 / 86.5 \pm 4.2 / 1.9 \mathrm{mmHg}$ in the drug group, and showed no significant difference. The systolic blood pressure was between 160 and $200 \mathrm{mmHg}$, but the diastolic blood pressure of $90 \mathrm{mmHg}$ or over was found in $56.0 \%$ of total subjects, $57.4 \%$ in the placebo group and $54.6 \%$ in the drug group. In laboratory data cholesterol levels were 175.4 $\mathrm{mg} / 100 \mathrm{ml}$ in the placebo group and $173.0 \mathrm{mg} / 100 \mathrm{ml}$ in the drug group. Blood urea nitrogens were 19.0 and $18.0 \mathrm{mg} / 100 \mathrm{ml}$, uric acids were 5.2 and $5.5 \mathrm{mg} / 100 \mathrm{ml}$, hemoglobin concentrations were 13.0 and $12.9 \mathrm{Gm} / 100 \mathrm{ml}$, cardiothoracic ratios in chest X-ray were 55.3 and 56.3\%, ECG scores for LVH were 1.1 and 1.3 respectively. By all factors measured the 2 groups were considered to be comparable.

Cerebro-cardiovascular and other complications during the 4 year trial period:

The cerebrovascular and cardiac complications observed during the 4 year prospective trial are shown in Table III. Six cases of dropout due to moving out from the Home were observed in both groups, and follow up cases were 38 in the drug group and 41 in the placebo group. The mortal events in the drug group were one each of cerebral infarction, cerebral hemorrhage and sudden death, and deaths in the placebo group were I case of cerebral infarction and 2 cases of myocardial infarction. The morbid events which needed hospital admission in the drug group were 1 case of cerebral infarction, and

Table II. Blood Pressure and Laboratory Data during the Control Period

\begin{tabular}{ll|r|r|r|r}
\hline & & \multicolumn{2}{|c|}{ Placebo } & \multicolumn{2}{|c}{ Drug } \\
\cline { 3 - 6 } & & mean & $\mathrm{SD}$ & mean & $\mathrm{SD}$ \\
\hline Blood pressure & systolic & 166.1 & 4.3 & 171.3 & 4.2 \\
(mmHg) & diastolic & 85.5 & 2.2 & 86.5 & 1.9 \\
Cholesterol & $\mathrm{mg} / 100 \mathrm{ml}$ & 175.4 & 29.6 & 173.1 & 30.9 \\
Urea nitrogen & $\mathrm{mg} / 100 \mathrm{ml}$ & 19.0 & 9.3 & 18.0 & 3.7 \\
Uric acid & $\mathrm{mg} / 100 \mathrm{ml}$ & 5.2 & 1.4 & 5.5 & 1.0 \\
Hemoglobin & $\mathrm{Gm} / 100 \mathrm{ml}$ & 13.0 & 1.2 & 12.9 & 1.8 \\
Cardiothoracic ratio & $\%$ & 55.3 & 6.2 & 56.3 & 6.2 \\
ECG scorc & $*$ & 1.1 & 0.9 & 1.3 & 0.9 \\
* Score for the diagnosis of the severity of hypertension, Department of Medicine, University \\
of Tokyo.
\end{tabular}


Table III. Cerebrovascular and Cardiac Complications during the 4 Year Prospective Trial

\begin{tabular}{l|c|c}
\hline & Placebo (41) & Drug (38) \\
\hline Death & 1 & 1 \\
cerebral infarction & 0 & 1 \\
cerebral hemorrhage & 2 & 0 \\
myocardial infarction & 0 & 1 \\
sudden death & 2 & 1 \\
Admission & 2 & 0 \\
cerebral infraction & 1 & 0 \\
CHF, arrhythmia & 1 & 0 \\
Outpatient & $9(22.0 \%)$ & $4 \quad(10.5 \%)$ \\
cerebral infarction & & \\
CHF, arrhythmia & &
\end{tabular}

Table IV. Complications during the 4 Year Prospective Trial

\begin{tabular}{|c|c|c|c|c|}
\hline & Placebo & \multicolumn{2}{|c|}{ Drug } & $\mathrm{p}$ \\
\hline Case & $47 \quad(41)$ & 44 & (38) & \\
\hline Move out & 6 & 6 & & \\
\hline Cerebrovascular and cardiac complications & & & & \\
\hline Death & 3) & 3) & & \\
\hline Admission & $4\} 9$ & $1\}$ & 4 & \\
\hline Outpatient & $2 \int(22.0 \%)$ & 0 & $(10.5 \%)$ & ns \\
\hline Elevated blood pressure & 8 & & & \\
\hline Total & $17 \quad(41.5 \%)$ & 4 & $(10.5 \%)$ & $\mathrm{p}<0.01$ \\
\hline Other complications & & & & \\
\hline Death & $4 \lcm{17}$ & $4 \mid$ & 12 & \\
\hline Admission & $13\}(41.5 \%)$ & 8 & $(31.6 \%)$ & ns \\
\hline Total complications & 34 & 16 & & \\
\hline Follow up & $7 \quad(17.1 \%)$ & 22 & $(57.9 \%)$ & $\mathrm{p}<0.001$ \\
\hline
\end{tabular}

in the placebo group 2 cases of cerebral infarction and 2 cases of congestive heart failure with arrhythmia were observed. The morbid events which were treated at outpatient deparment were one each of cerebral infarction and congestive heart failure with arrhythmia. As a whole 9 out of 41 cases or $22.0 \%$ in the placebo group, and 4 out of 38 cases or $10.5 \%$ in the drug group dropped out by cerebrovascular or cardiac complications. This difference was not significant statistically. In addition to the cerebrovascular and cardiac complications dropouts due to blood pressure elevation were observed in 8 cases in the placebo group, and total dropouts in the placebo group were 17 cases or $41.5 \%$. This incidence was significartly higher than that in the drug treat- 
Table V. Other Miscellaneous Complications during the 4 Year Trial

\begin{tabular}{l|c|c|c}
\hline & Placebo (41) & & Drug (38) \\
\hline Death & 4 & & 4 \\
cancer & 1 & & 4 \\
esophagus & 1 & stomach & 2 \\
& & lung & 1 \\
pneumonia & 2 & gall bladder & 1 \\
milliary tbc & 1 & & 0 \\
Admission & 13 & & 0 \\
arthrosis deformans & 1 & & 8 \\
spondylosis deformans & 1 & & 2 \\
fracture of femor neck & 3 & & 2 \\
dementia, psychosis & 3 & prostate & 0 \\
cancer $\quad$ stomach & 2 & urinary tract infection & 1 \\
prostate hypertrophy & 1 & inguinal hernia & 1 \\
arteriosclerosis obliterans & 1 & glaucoma & 1 \\
pneumonia & 1 & & 12 \\
Total & 17 & & \\
& & & \\
& & & \\
\end{tabular}

ed group (Table IV).

Other complications were observed in 12 cases or $31.6 \%$ in the drug group, and in 17 cases or $41.5 \%$ in the placebo group. These were not different significantly. Therefore, cases without events were 22 cases or $57.9 \%$ in the drug group, and 7 cases or $17.1 \%$ in the placebo group showing a significant difference. Other complications are shown in Table V. Four deaths in the drug group were due to cancers of the stomach, lung and gall bladder, and 4 deaths in the placebo group were caused by pneumonia, miliary tuberculosis, and cancer of the esophagus. Morbid events were observed in 8 cases in the drug group and in 13 cases in the placebo group. Major causes for admission in these patients were bone and joint diseases, cancer, psychiatric disorder, infections, prostate hypertrophy, and others. It should be noticed that these events were 2 or 3 times higher than that of the cerebrovascular and cardiac complications.

Course of blood pressure in the drug and placebo groups:

The trends of mean blood pressure at each month are shown in Fig. 1. The average blood pressure in the drug group was lowered from 171/87 to $162 / 84$ in the first year, 156/80 in the second year, 152/80 in the third year and $151 / 80$ in the fourth year. The average decrease was $20 / 7 \mathrm{mmHg}$. The 


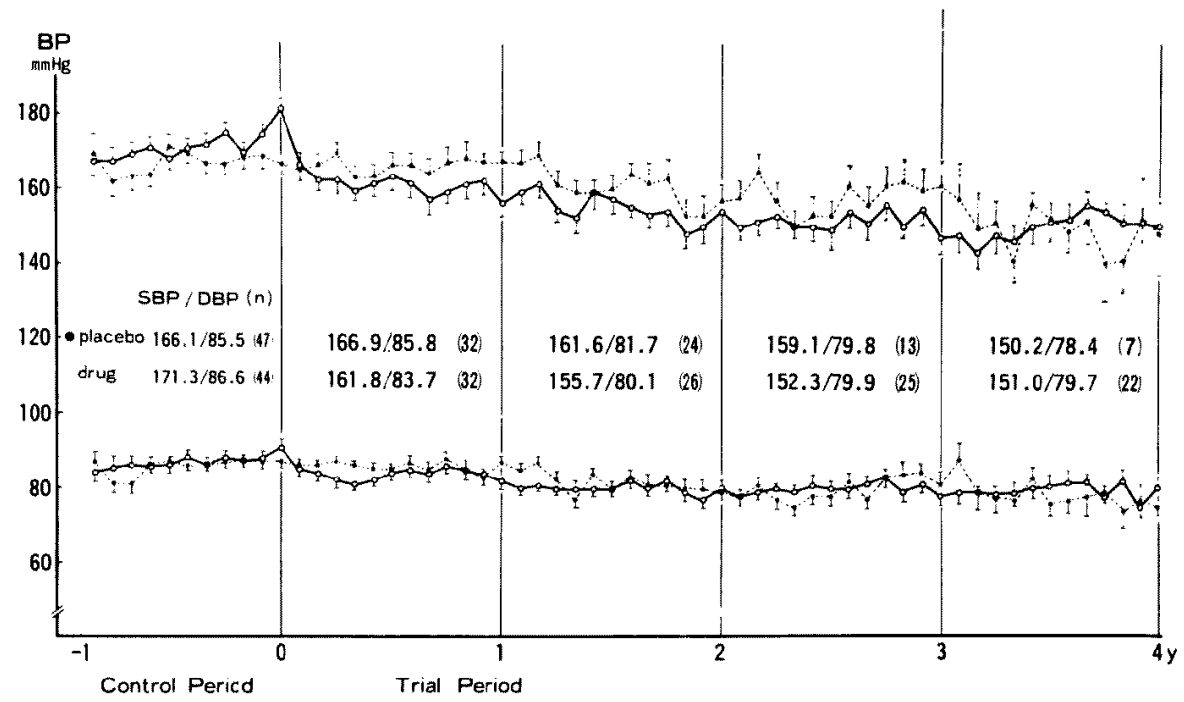

Fig. 1. Course of blood pressure during the control and trial period in the total cases of drug and placebo groups.

number of the follow up cases at the end of each year declined from 44 to 32 , 26,25 , and 22 due to dropouts. Blood pressure in the placebo group changed little from $166 / 86$ to $167 / 86$ in the first year, but gradually declined to $162 / 82$ in the second year, 159/80 in the third year, and 150/78 in the fourth year due to dropouts of the hypertensive cases. The number of the follow up cases in the placebo group decreased markedly from 47 to $32,24,13$, and 7 at the end of each year. In 22 cases of the drug group followed up through 4 year trial period blood pressure declined from $171 / 87$ to $162 / 84,154 / 80,152 / 80$, and $150 / 79$ in each year, and the average decrease was $21 / 8 \mathrm{mmHg}$ (Fig. 2). In 7 cases of placebo group the blood pressure during the control period was relatively low and gradually declined in the placebo period probably due to aging.

The blood pressures before the onset of cerebrovascular or cardiac complications in the drug treated patients oscillated arround 140/80, and no abrupt changes were observed. Blood pressures in the 9 placebo treated patients were fluctuated in the higher level, but no trend of elevation was observed.

\section{Laboratory data during the trial period:}

Changes in laboratory data in 16 thiazide trated patients and 11 placebo treated patients are shown in Fig. 3. Hematocrit, serum protein, urea nitrogen, uric acid, sodium, and potassium for 3 year trial period revealed no sign- 


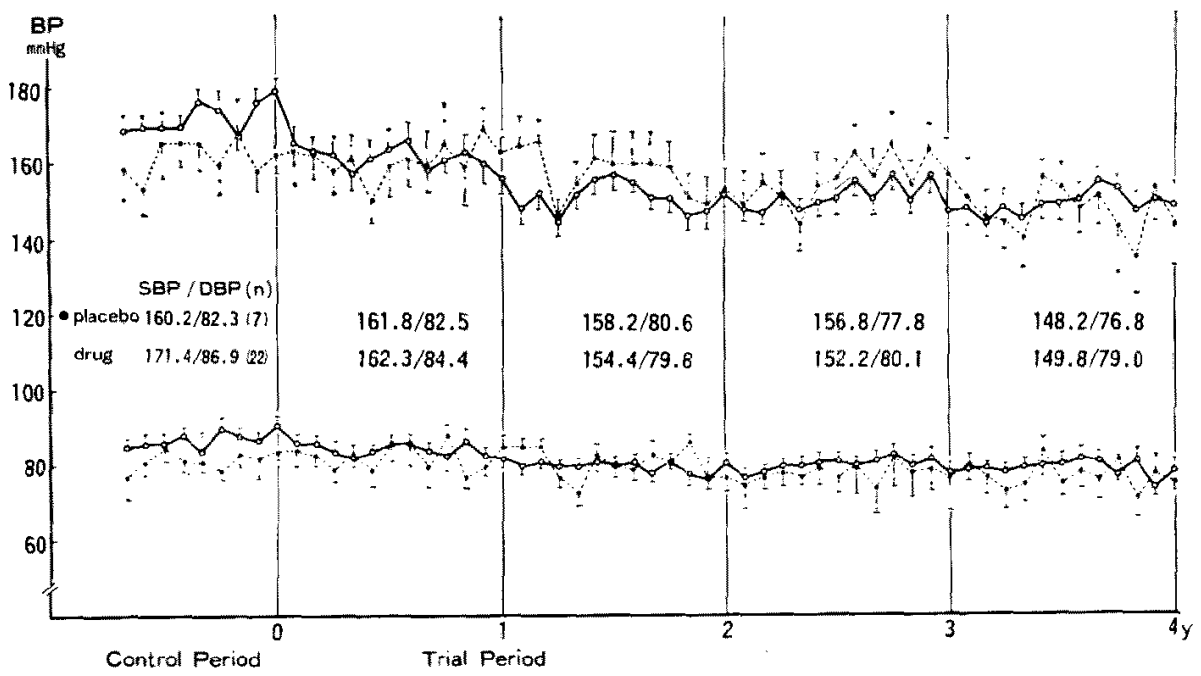

Fig. 2. Course of blood pressure in 7 cases of placebo group and 22 cases of drug group followed up through 4 year trial period.
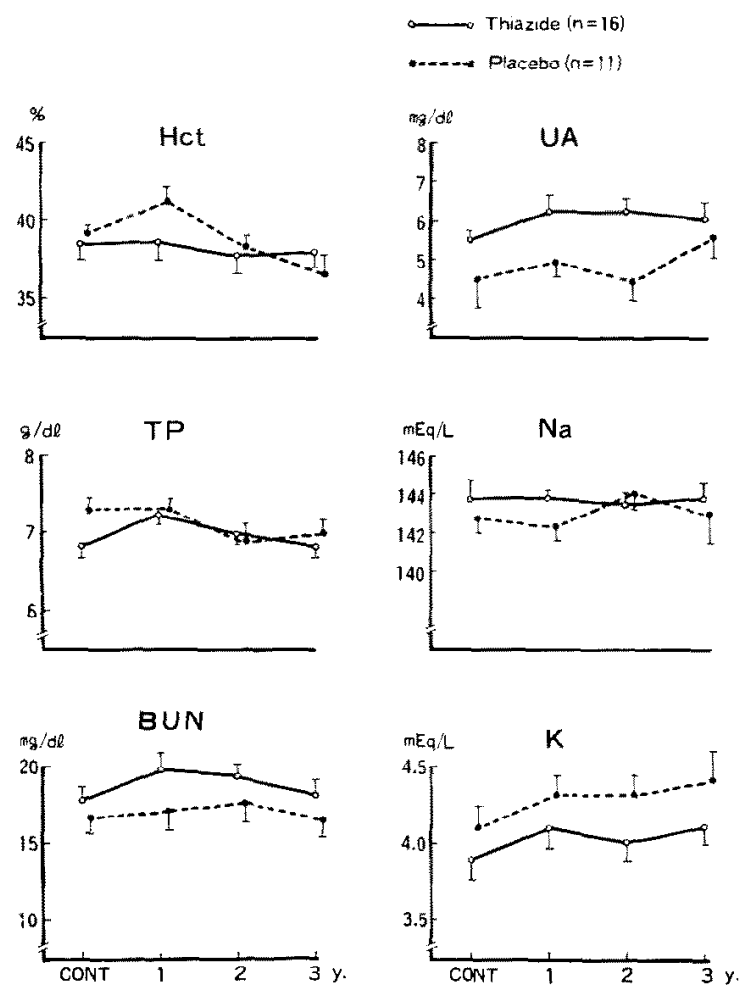

Fig. 3. Laboratory data during the trial period in thiazide and placebo groups. 
igicant changes. The uric acid in thiazide treated group was slightly elevated from 5.5 to $6.2 \mathrm{mg} / 100 \mathrm{ml}$, but not different signigicantly.

\section{Discussion}

The present prospective study revealed that antihypertensive treatment in the elderly with mild hypertension was effective in decreasing the cerebrovascular and cardiac complications when elevation of blood pressure was counted as a dropout. The cerebrovascular and cardiac complications were observed in $10.5 \%$ in the drug treated group, and in $22.0 \%$ in the placebo group. This difference was not significant probably due to the small number of the cases. When blood pressure elevation in the placebo group were added to the complications, dropouts in the placebo group reached $41.5 \%$. This showed the significant difference. However, other complications such as cancers, bone or joint diseases, infections, psychiatric disorders were 2 or 3 times more prevalcnt than cerebro-cardiovascular complications.

There has been no general agreement on the effect of the antihypertensive treatment in the elderly. The negative aspect on the antihypertensive therapy was propsed by Fry. ${ }^{11}$ He observed the natural history of hypertension and found no increasing mortality rates with rising blood pressure in the over-sixties. Carter ${ }^{2)}$ followed up 99 cases of diastolic pressure over 110 $\mathrm{mmHg}$ for 2 to 5 years, and found the stroke recurrence to be $44 \%$ in placebo group, and $20 \%$ in drug terated group, but found no beneficial effect on the mortality over the age of 65 . Jackson et $\mathrm{al}^{3)}$ presented the 6 symptomless hypertensive cases aged 64 to $\mathbf{8 4}$ who revealed unconsciousness and residual symptoms after the inappropriate antihypertensive therapy, and emphasized that the potent therapy might be unnecessary or harmful in the aged.

The prospective study on the antihypertensive therapy in patients above 60 years was carried out by the European Working Party on High blood pressure in the Elderly (EWPHE). ${ }^{7}$ They observed $25 / 10 \mathrm{mmHg}$ reduction of the blood pressure in 2 years in the absence of major electrolyte disturbances. But the terminating events were still being followed to prove the difference. Terasawa ${ }^{8)}$ carried out the prospective study on 121 hypertensive patients in the aged. The incidences of cerebrovascular disease, congestive heart failure and myocardial infarction in 5 year period were $17.1 \%$ in the drug group and $31.4 \%$ in the placebo group showing no significant difference. The incidence of morbid events in the drug group was similar to the present results, but that of the placebo group was higher than our data probably due to the difference of protocol in treating cases with blood pressure elevation. Svärdsudd et al ${ }^{9}$ followed up hypertensive patients of 50 years old for 13.5 years and concluded 
that hypertension accerelates cerebrovascular and cardiac events. The usefulness of antihypertensive treatment in mild hypertension was proved in the VA study in cases of diastolic blood pressure of 90 to $114 \mathrm{mmHg} .{ }^{10}$ ) The incidence of morbid events was $11.8 \%$ in the drug group and $28.9 \%$ in the placebo group. When the cases terminated on account of elevated blood pressure in the placebo group were summed up with the morbid events, total assessable events reached $39.2 \%$. Since the average ages in the VA study were 52.0 and 50.5 years, these effects were not applicable to the aged. However, the prevalences of morbid events were fairly similar to the present study in the aged. The VA study analysed the morbid events in different age gronps. ${ }^{11}$ The incidences of morbid events in the fifties were $8.7 \%$ in the treated group and $26.9 \%$ in the control group, and these incidences over the sixties were $28.9 \%$ and $62.9 \%$ respectively showing the effectiveness of antihypertensive treatment in the aged even in the group with lower diastolic pressure of 90 to $104 \mathrm{mmHg}$. The study by the Society of Actuaries concluded that more deaths could occur from untreated hypertension in the older population, and hypertensives with onset at age 35 had 15 excess death in the subsequent 20 years, but those with onset at age 55 had 25 excess death in the same interval. ${ }^{12}$ ) The USPHS study ${ }^{13)}$ on the treatment of mild hypertension of younger population revealed no significant difference between the treated and control groups. The treatment of the mild hypertension in younger population should be directed to other risk factors or general health control. The mild hypertension in the aged should be treated, since the hypertension would trigger cardiovascular events on the basis of atherosclerosis of the target organs.

Systolic hypertension was frequently observed in the elderly, consisting $44 \%$ of the present subjects. Colandrea et $\mathrm{al}^{4}{ }^{4}$ found that cardiovascular complications were more prevalent in systolic hypertension than in normotension in the aged of 69 years on average. Kannel et $\mathrm{al}^{5 /}$ revealed a positive relationship between systolic hypertension and cerebral infarction or coronary heart diseases, and emphasized the need for the treatment of systolic hypertension. Kuramoto et $\mathrm{al}^{14}$ ) found the atherosclerosis of cerebral and coronary arteries as well as cerebovascular and coronary complications in systolic hypertension to be similar to that in diastolic hypertension, and significantly different from normotensives in the aged.

On the basis of the present study we concluded that antihypertensive treatment in mild hypertension of the aged was effective to prevent cerebrovascular and cardiac events if the patients revealed normal activity of daily living and social activities. However, the treatment should follow the stepped care approach $^{15}$ ) with a small starting dosis, and should not lower the blood pressure abruptly. The target level of blood pressure should be determined 
individually watching the general health condition of the patients. The blood pressure in the drug group of the present study reached around 150/80 $\mathrm{mmHg}$. Taguchi and Freis ${ }^{16)}$ repoted that the partial reduction of blood pressure in VA study was still effective in prevention of morbid events. The cardiovascular complications were of $14.9 \%$ and $9.7 \%$ in groups with posttreatment diastolic blood pressures of $90 \mathrm{mmHg}$ or higher and $80 \mathrm{mmHg}$ or less, and these attack rates were significantly less than the $28.9 \%$ of patients in the placebo group.

The present study used a small amount of thiazide diuretics (trichlormethiazide 1 to $4 \mathrm{mg}$ ) as a basal antihypertensive drug, and found no significant changes in potassium, uric acid, hematocrit and others. However, care should be taken on the electrolytes, blood sugar, uric acid, blood urea nitrogen, and hematocrit to prevent the specific side effect in the elderly. The morbid events in the elderly hypertension were not only cerebrovascular or cardiac complications but also other complications such as bone and joint diseases, cancer, infection, psychiatric disorder and others, and the other complications were 2 or 3 times more prevalent than the cerebrovascular or cardiac complications indicating the need for the general health ckeck up during the antihypertensive treatment.

\section{REFERENCES}

1. Fry J: Natural history of hypertension, a case for selective non-treatment. Lancet 2: 431, 1974

2. Carter AB: Hypotensive therapy in stroke survivors. Lancet 1: 485, 1970

3. Jackson $G$, Pierscianowski TA, Mahon $W$ et al: Inappropriate antihypertencive therapy in the elderly. Lancet 2: 1317, 1976

4. Colandrea MA, Friedman GD, Nichaman MS et al: Systolic hypertension in the elderly: An epidemiologic assessment. Circulation 41: 239, 1970

5. Kannel WB, Gordon T, Schwartz MJ: Systolic versus diastolic blood pressure and risk of coronary heart disease. The Framingham study. Am J Cardiol 27: 335, 1971

6. Kuramoto K, Seki A, Matsushita S et al: Blood pressure elevation after withdrawal of antihypertensive drugs in the aged. Jpn J Geriat 15: 562, 1978

7. Amery A, Berthaux P, Birkenhager $W$ et al: Antihypertensive therapy in patients above age 60. Acta Cardiol 33: 113, 1978

8. Terasawa F: Antihypertensive treatment in the aged. Jpn J Geriat 12: 235, 1975 (in Japanese)

9. Svärdsudd $\mathrm{K}$, Tibblin G: Mortality and morbidity during 13.5 years' follow-up in relation to blood pressure. The study of men born in 1913. Acta Med Scand 205: 483, 1979

10. Veterans Administration Cooperative Study Group on Antihypertensive Agents: Effects of treatment on morbidity in hypertension II. JAMA 213: 1143, 1970

11. Veterans Administration Cooperative Study Group on Antihypertensive Agents: Effects of treatment on morbidity in hypertension III. Influence of age, diastolic pressure, and prior cardiovascular disease; further analysis of side effects. Circulation 45: 991, 1972

12. Neutra R: Treatment of hypertension. Lancet 2: 1089, 1974 
13. Smith WM: Treatment of mild hypertension. Results of a ten-year intervention trial. Circulat Res 40 (Suppl): 1-98, 1977

14. Kuramoto $\mathrm{K}$, Matsushita $\mathrm{S}$, Kuwajima I: The pathogenesis and treatment in elderly hypertension. Jpn Circulat J 45: 1981 (in preparation)

15. AMA Committee on Hypertension: Drug treatment of ambulatory patients with hypertension. JAMA 225: 1647, 1973

16. Taguchi J, Freis ED: Partial reduction of blood pressure and prevaention of complications in hypertension. New Engl J Med 291 : 329, 1974 